\title{
Hubble Volume and the Fundamental Interactions
}

\author{
U. V. S. Seshavatharam ${ }^{1, *}$, S. Laks hminarayana ${ }^{2}$ \\ ${ }^{1}$ Honorary faculty, I-SERVE, Alakapuri, Hyderabad, 35, AP, India \\ ${ }^{2}$ Dept. of Nuclear Phy sics, Andhra University, Visakhapatnam, 03, AP, India
}

\begin{abstract}
In modern cosmology, the shape of the universe is flat. In between the closed space and flat space, there is one compro mise. That is 'Hubble volu me'. Even though Hubble volume is virtual in the flat universe, by considering the product of critical mass density and the Hubble volume, one can estimate the Hubble mass. By coupling the Hubble mass with the Mach's principle, one can understand the origin of cosmic, atomic and nuclear physical parameters. Thus the four fundamental interactions can be studied in a unified manner.
\end{abstract}

Keywords Hubble Radius, Hubble Volume, Hubble Mass, Mach's Principle, Planck Mass, Coulomb Mass, Fine Structure Ratio, the 4 Fundamental Interactions, SUSY and CMBR Temperature

\section{Introduction}

In 1998, published observations of Type Ia supernovae by the High-z Supernova Search Team followed in 1999 by the Supernova Cosmology Project suggested that the expansion of the universe is accelerating. 2011 Nobel Prize in Physics was awarded for this work. According to the WMAP seven-year analys is, universe cons titutes $72.8 \%$ dark energy, $22.7 \%$ dark matter and $4.6 \%$ ordinary matter. Authors would like to ask the questions: What are the important applications of the $72.8 \%$ dark energy or $22.7 \%$ matter in the other important fundamental areas of physics (like unification of the fundamental interactions)? What is the role of dark matter or dark energy in Hydrogen atom or the atomic nucleus? To find a way to answer such questions, in this paper authors made an attempt to combine and study the concepts of 'cosmic critical density', 'Hubble volume' and the 'Mach's principle' in a unified semi emp irical approach.

Please note that, when it was proposed in 1948, the CMBR idea was never accepted by the science community. But, in 1965 , this concept was realized serendipitously. The very surprising thing was that the experimentalists were not aware of what they discovered! Up to 1998, people believed in cosmic deceleration. By 2000, it was a shocking news to many cosmologists that, the universe is accelerating. Please note that, still some scientists argue that, the only indication for the existence of dark energy is observations of distance measurements and associated redshifts. Cosmic microwave background anis otropies and baryon acoustic oscillations are only observations that redshifts are larger than expected from a "dusty" Friedmann-Lemaitre universe and the local

* Corresponding author:

seshavatharam.uvs@gmail.com (U. V. S. Seshavatharam)

Published online at http://journal.sapub.org/astronomy

Copyright (C) 2012 Scientific \& Academic Publishing. All Rights Reserved measured Hubble constant.

Here it is very important to note that, in reality no one measured the galaxy's receding speed! But it is the required primary measurement. Based on the Hubble's law, as a secondary or indirect measurement, receding galaxy's redshift is being measured. This is the normal practice and in support of that, galaxy's estimated distance is compared with other secondary methods! If the universe is really accelerating, based on the same Hubble's law, for the observer - the receding and accelerating galaxy must show a continuous increase in its red shift! Some says: instantaneously red shift cannot increase due to the limited photon speed. If cosmic acceleration began 5 billion years ago, then during its accelerated receding journey, the galaxy must show a continuous increase in red shift - whether the change is due to past accelerated receding or present accelerated receding. There is no such evidence. In this connection - the appropriate idea can be stated as follows: 'rate of increase in red shift is a measure of cosmic rate of expansion'. This idea can be supported by another interesting concept: 'rate of decrease in CMBR temperature is a measure of cosmic rate of expansion'.

\subsection{Current Status of Mach's Principle - Hubble Volume}

In theoretical physics, particularly in discussions of gravitation theories, Mach's principle[1-6] is the name given by Einstein to an interesting hypothes is often credited to the physicist and philosopher Ernst Mach. The idea is that the local motion of a rotating reference frame is determined by the large scale distribution of matter. There are a number of rival formulations of the princip le. A very general statement of Mach's principle is 'local physical laws are determined by the large-scale structure of the universe'. This concept was a guiding factor in Einstein's development of the general theory of relativity. Einstein realized that the overall distribution of matter would determine the metric tensor, 
which tells the observer which frame is rotationally stationary.

One of the main motivations behind formulating the general theory of relativity was to provide a mathematical description to the Mach's principle. However, soon after its formulation, it was realized that the theory does not follow Mach's principle. As the theoretical predictions were matching with the observations, Einstein believed that the theory was correct and did not make any farther attempt to reformu late the theory to explain Mach's principle. Later on, several attempts were made by different researchers to formulate the theory of gravity based on Mach's principle. However most of these theories remain unsuccessful to explain different physical phenomena.

In the standard cosmology, "Hubble volume" or "Hubble sphere" is a spherical region of the Universe surrounding an observer beyond which objects recede fro $m$ that observer at a rate greater than the speed of light due to the expansion of the Universe. The commoving radius of a Hubble sphere (known as the Hubble radius or the Hubble length) is, $\left(c / H_{0}\right)$, where $(c)$ is the speed of light and $\left(H_{0}\right)$ is the Hubble constant. More generally, the term "Hubble volume" can be applied to any region of space with a volume of the order of $4 \pi / 3\left(c / H_{0}\right)^{3}$.

\subsection{Proposed New Concepts on the Mach's Principle, Hubble Volume and Hubble Mass}

Note that till today quantitatively Mach's principle was not implemented successfully in cosmic and nuclear physics. If we do not yet know whether the universe is spatially closed or open, then the idea of Hubble volume can be used as a tool in cosmology and unification. Considering the particle and event horizon concepts, where ever we go in the flat universe, for the observer, Hubble volume is the only observable/workable volume. Hence where ever we go in the universe, Hubble volu me plays the same role. It seems to be a quantitative description to the Mach's principle. In the universe, if the critical density is $\rho_{c} \cong\left(3 H_{0}^{2} / 8 \pi G\right)$ and the characteristic Hubble radius is $R_{0} \cong\left(c / H_{0}\right)$, mass of the cosmic Hubble volume is $M_{0} \cong \frac{c^{3}}{2 G H_{0}}$. For the time being let us call this mass as "Hubble mass". With this definition, apart from cosmology, Mach's principle can be given a fundamental unified significance in atomic, nuclear and particle physics! Here, as a point of curiosity, if one is willing to consider this mass as a characteristic mass of the universe, very easily, planck scale, cos mology and particle physics can be studied in a unified manner. It depends only on our choice of scientific interest. If $m_{p}$ is the rest mass of proton and $m_{e}$ is the rest mass of electron, it is noticed that,

$$
R_{s} \cong \frac{G \sqrt{M_{0} \sqrt{m_{p} m_{e}}}}{c^{2}} \cong(1.37 \text { to } 1.39) \times 10^{-15} \mathrm{~m}
$$

This observed length can be compared with Yukawa's strong interaction range or the characteristic nuclear unit radius.

$$
2 R_{s} \cong \frac{2 G \sqrt{M_{0} \sqrt{m_{p} m_{e}}}}{c^{2}} \cong(2.74 \text { to } 2.78) \times 10^{-15} \mathrm{~m}
$$

This is close to the classical radius of electron! If $M_{p}$ is the Planck mass and $R_{0} \cong\left(c / H_{0}\right)$ is the gravitational and electro magnetic interaction range, it is noticed that,

$$
\ln \left(\frac{m_{e} R_{0}^{2}}{M_{P} R_{s}^{2}}\right) \cong \frac{1}{137.2} \cong \frac{1}{\alpha} .
$$

This is another interesting coincidence! How to interpret these relations? Here, the utmost fundamental observation is: all the be lieved ato mic and nuclear constants are join ing with the growing cosmic Hubble size or Hubble volume or the Hubble mass. In the accelerating universe, how is it possible? From these relations it is possible to say that: 1) In the Hubble volume, each and every point in the free space is influenced by the Hubble mass, 2) Within the Hubble volume, the Hubble mass (distribution) plays a vital role in understanding the properties of electro magnetic and nuclear interactions and 3) Hubble mass plays a key role in understanding the geometry of the universe.

Including the $\mathrm{CMB}$ radiation energy density \& the observed matter- energy density, in this connection, authors observed so many interesting relations. Whether this is the beginning of a controversy or the beginning of unification, for the time being authors propose the following (interesting) observations and concepts related to Mach's principle, Hubble volume and the fundamental interactions. Also please see appendix 1 and 2. Appendix-1 is: 'The characteristic nuclear radii in Cosmology' and appendix-2 is : 'Cos mic critical density, matter density and thermal energy density'.

\section{Avogadro Number, Atom and the Universe}

The subject of unification is very interesting and very complicated[7-18]. By implementing the Avogadro number $N$ as a scaling factor in unification program, one can probe the constructional secrets of elementary particles. The Planck's quantum theory of light, thermodynamics of stars, black holes and cosmology totally depends upon the famous Boltzmann constant $k_{B}$ which in turn depends on the Avogadro number[19]. From this it can be suggested that, Avogadro number is more fundamental and characteristic than the Boltzmann constant and indirectly plays a crucial role in the formulation of the quantum theory of radiation. In this connection it is noticed that, 'molar electron mass' plays a very interesting role in nuclear and part icle physics. Even if Avogadro number is a man-made number, authors humble opinion is - first let us find the various applications of the Avogadro number in unification. At any one nice relation, its meaning can be understood. The ratio of Planck mass and electron rest mass is close to Avogadro number/ $8 \pi$. This is a 
very interesting and surprising result.

\subsection{Key Concepts in Unification}

Concept-1: In the expanding cosmic Hubble volume, characteristic cosmic Hubble mass is the product of the cosmic critical density and the Hubble volume. If the critical density is $\rho_{c} \cong\left(3 H_{0}^{2} / 8 \pi G\right)$ and characteristic Hubble radius is $R_{0} \cong\left(c / H_{0}\right)$, mass of the cosmic Hubble volume is

$$
M_{0} \cong \frac{c^{3}}{2 G H_{0}}
$$

Concept-2: There exists a charged heavy massive elementary particle $M_{X}$ in such a way that, inverse of the fine structure ratio is equal to the natural logarithm of the sum of number of positively and negatively charged $M_{X}$ in the Hubble volume. If the number of positively charged $\left(M_{X}\right)^{+}$is $\left(\frac{M_{0}}{M_{X}}\right)$ and the number of negatively charged

$$
\begin{aligned}
\left(M_{X}\right)^{-} \text {is also } & \left(\frac{M_{0}}{M_{X}}\right) \text { then } \\
& \frac{1}{\alpha} \cong \ln \left(\frac{M_{0}}{M_{X}}+\frac{M_{0}}{M_{X}}\right) \cong \ln \left(\frac{2 M_{0}}{M_{X}}\right)
\end{aligned}
$$

From experiments $1 / \alpha \cong 137.0359997$ and from the current observations [20,21,22], magnitude of the Hubble constant is, $H_{0} \cong 70.4_{-1.4}^{+1.3} \mathrm{Km} / \mathrm{sec} / \mathrm{Mpc}$. Thus

$$
\begin{aligned}
M_{X} & \cong e^{-\frac{1}{a}}\left(\frac{c^{3}}{G H_{0}}\right) \cong e^{\frac{1}{\alpha}} \cdot 2 M_{0} \\
& \cong(5.32 \text { to } 5.53) \times 10^{-7} \mathrm{Kg}
\end{aligned}
$$

If $N \cong 6.022141793 \times 10^{23}$ is the Avogadro number and $m_{e}$ is the rest mass of electron, surprisingly it is noticed that, $N . m_{e} \cong 5.485799098 \times 10^{-7} \mathrm{Kg}$ and this is close to the above estimation of $M_{X}$. Thus it can be suggested that,

$$
\frac{M_{X}}{m_{e}} \cong N
$$

In this way, Avogadro number can be coupled with the cosmic, ato mic and particle physics. Then with reference to $\left(N . m_{e}\right)$, the obtained cosmic Hubble mass is $M_{0} \cong 8.957532458 \times 10^{52} \mathrm{Kg}$ and thus the obtained Hubble's constant is $H_{0} \cong \frac{c^{3}}{2 G M_{0}} \cong 69.54 \mathrm{Km} / \mathrm{sec} / \mathrm{Mpc}$. Note that large dimensionless constants and compound physical constants reflects an intrinsic property of nature $[23,24]$. Whether to consider them or discard them depends on the physical interpretations, logics, experiments, observations and our choice of scientific interest. In most of the critical cases, 'time' only will decide the issue. The mystery can be resolved only with further research, analys is, discussions and encouragement.
Concept-3: For any observable charged particle, there exists 2 kinds of masses and their mass ratio is 295.0606339. Let this number be represented by $X_{E}$. First kind of mass seems to be the 'gravitational or observed' mas $s$ and the second kind of mass seems to be the 'electro magnetic' mass. This idea can be applied to proton and electron.

This number is obtained in the following way. In the Planck scale, similar to the Planck mass, with reference to the elementary charge, a new mass unit can be constructed in the following way.

$$
\begin{aligned}
& M_{C} \cong \sqrt{\frac{e^{2}}{4 \pi \varepsilon_{0} G}} \cong 1.859210775 \times 10^{-9} \mathrm{Kg} \\
& M_{C} c^{2} \cong \sqrt{\frac{e^{2} c^{4}}{4 \pi \varepsilon_{0} G}} \cong 1.042941 \times 10^{18} \mathrm{GeV}
\end{aligned}
$$

Here $e$ is the elementary charge. How to interpret this mass unit? Is it a primordial massive charged particle? If 2 such oppositely charged particles annihilates, a large amount of energy can be released. Considering so many such pairs annihilation hot big bang or in flation can be understood. This may be the root cause of cosmic energy reservoir. Such pairs may be the chief constituents of black holes. In certain time interval with a well defined quantum rules they annihilate and release a large amount of energy in the form of $\gamma$ photons. In the Hubble volume, with its pair annihilation, origin of the CMBR can be understood. Clearly speaking, gravitational and electromagnetic force ratio of $M_{X}$ is $X_{E}^{2}$.

$$
\frac{M_{X}}{M_{C}} \cong \sqrt{\frac{4 \pi \epsilon_{0} G M_{X}^{2}}{e^{2}}} \cong 295.0606338
$$

It can be interpreted that, if $5.486 \times 10^{-7} \mathrm{Kg}$ is the observable or gravitational mass of $M_{X}$, then $M_{C}$ is the electromagnetic mass of $M_{X}$.

With reference to the electron rest mass,

$$
\left(\frac{M_{X}}{m_{e}}\right)^{2} \cong X_{E}^{2} \cdot \frac{e^{2}}{4 \pi \epsilon_{0} G m_{e}^{2}} \cong N^{2}
$$

Concept-4: If $\hbar$ is the quantum of the gravitational angular momentum, then the electromagnetic quantum can be expressed as $\left(\frac{\hbar}{X_{E}}\right)$. Thus the ratio,

$$
\left(\frac{\hbar}{X_{E}}\right) \div\left(\frac{e^{2}}{4 \pi \epsilon_{0} c}\right) \cong\left(X_{E} \alpha\right)^{-1} \cong 0.464433353 \cong \sin \theta_{W}
$$

where $\sin \theta_{W}$ is very close to the weak mixing angle

Concept-5: In modified quark SUSY[25], if $Q_{f}$ is the mass of quark fermion and $Q_{b}$ is the mass of quark boson, then

$$
\frac{m_{f}}{m_{b}} \cong \Psi \cong 2.2627062
$$

and $\left(1-\frac{1}{\Psi}\right) Q_{f}$ represents the effective fermion mass. The number $\Psi$ can be fitted with the following empirical 
relation

$$
\Psi^{2} \ln \left(1+\sin ^{2} \theta_{W}\right) \cong 1
$$

With this idea super symmetry can be observed in the strong interactions [25] and can also be observed in the electroweak interactions[26-28].

Concept-6: For electron, starting from infinity, its characteristic interaction ending range can be expressed as

$$
r_{e e} \cong \frac{e^{2}}{4 \pi \epsilon_{0}\left(m_{e} / X_{E}\right) c^{2}} \cong X_{E} \frac{e^{2}}{4 \pi \epsilon_{0} m_{e} c^{2}} \cong 8.315 \times 10^{-13} \mathrm{~m}
$$

Similarly, for proton, its characteristic interaction starting range can be expressed as

$$
r_{s s} \cong \frac{e^{2}}{4 \pi \epsilon_{0}\left(m_{p} / X_{E}\right) c^{2}} \cong X_{E} \frac{e^{2}}{4 \pi \epsilon_{0} m_{p} c^{2}} \cong 4.53 \times 10^{-16} \mathrm{~m} \text { (13) }
$$

Concept-7: Ratio of electromagnetic ending interaction range and strong interaction ending rage[29] can be expressed as

$$
\frac{r_{e e}}{r_{s e}} \cong \frac{G M_{X}^{2}}{\hbar c} \cong 635.3131866
$$

Thus if $r_{e e} \cong 8.315 \times 10^{-13} \mathrm{~m}, r_{s e} \cong 1.309 \times 10^{-15} \mathrm{~m}$,

$$
\left(\frac{r_{e e}}{r_{s e}}\right)^{2} \cong\left(\frac{G M_{X}^{2}}{\hbar c}\right)^{2}
$$

Interesting observation is

$$
\frac{r_{s S}+r_{s e}}{2} \cong 0.881 \times 10^{-15} \mathrm{~m}
$$

This can be considered as the mean strong interaction range and is close to the proton rms radius[30]!

Concept-8: For any elementary particle of charge $e$, electro magnetic mass $\left(m / X_{E}\right)$ and characteristic radius $R$, it can be assumed as

$$
\frac{e^{2}}{4 \pi \epsilon_{0} R} \cong \frac{1}{2}\left(\frac{m}{X_{E}}\right) c^{2}
$$

This idea can be applied to proton as well as electron. Electron's characteristic radius is

$$
R_{e} \cong 2 X_{E} \frac{e^{2}}{4 \pi \epsilon_{0} m_{e} c^{2}} \cong 1.663 \times 10^{-12} \mathrm{~m}
$$

Similarly proton's characteristic radius is

$$
R_{p} \cong 2 X_{E} \frac{e^{2}}{4 \pi \epsilon_{0} m_{p} c^{2}} \cong 0.906 \times 10^{-15} \mathrm{~m}
$$

This obtained magnitude can be compared with the rms charge radius of the proton[30]. W ith different experimental methods its magnitude varies from $0.84184(67) \mathrm{fm}$ to $0.895(18) \mathrm{fm}$.

\subsection{Potential Energy of Electron In Hydrogen Atom}

Let $E_{p}$ be the potential energy of electron in the Hydrogen atom. It is noticed that,

$$
E_{p} \cong \frac{e^{2}}{4 \pi \epsilon_{0} a_{0}} \cong\left(\frac{\hbar c}{G M_{X}^{2}}\right) \frac{\left(\hbar / X_{E}\right) c}{\sqrt{R_{e} R_{p}}} \cong 27.12493044 \mathrm{eV}
$$

where $a_{0}$ is the Bohr radius[31,32]. With $99.6822 \%$ this is matching with $\alpha^{2} m_{e} c^{2} \cong 27.21138388 \mathrm{eV}$. After simplification it takes the following form.

$$
E_{p} \cong\left(\frac{\hbar c}{G M_{X}^{2}}\right)^{2} \frac{\sqrt{m_{p} m_{e}} c^{2}}{2} \cong \alpha^{2} m_{e} c^{2}
$$

Thus the Bohr radius can be expressed as

$$
a_{0} \cong\left(\frac{G M_{X}^{2}}{\hbar c}\right)^{2} \frac{2 e^{2}}{4 \pi \epsilon_{0} \sqrt{m_{p} m_{e}} c^{2}}
$$

Without considering the integral nature of angular momentum, here by considering the integral nature of the elementary charge $e$, Bohr radius in $n^{\text {th }}$ orbit can be expressed as

$$
a_{n} \cong\left(\frac{G M_{X}^{2}}{\hbar c}\right)^{2} \frac{2(n e)^{2}}{4 \pi \epsilon_{0} \sqrt{m_{p} m_{e}} c^{2}} \cong n^{2} \cdot a_{0}
$$

where $a_{n}$ is the radius of $n^{\text {th }}$ orbit and $n=1,2,3, .$. Thus in Hydrogen atom, potential energy of electron in $n^{\text {th }}$ orbit can be expressed as

$$
\frac{e^{2}}{4 \pi \epsilon_{0} a_{n}} \cong\left(\frac{\hbar c}{G M_{X}^{2}}\right)^{2} \frac{\sqrt{m_{p} m_{e}} c^{2}}{2 n^{2}}
$$

Note that, from the atomic theory it is well established that, total number of electrons in a shell of principal quantum number $n$ is $2 n^{2}$. Thus on comparison, it can suggested that, $\left(\frac{\hbar c}{G M_{X}^{2}}\right)^{2} \sqrt{m_{p} m_{e}} c^{2}$ is the potential energy of $2 n^{2}$ electrons and potential energy of one electron is equal to $\left(\frac{\hbar c}{G M_{X}^{2}}\right)^{2} \frac{\sqrt{m_{p} m_{e}} c^{2}}{2 n^{2}}$.

\subsection{Magnetic Moments of the Nucleon}

If $\left(\alpha X_{E}\right)^{-1} \cong \sin \theta_{W}$, magnetic moment of electron can be expressed as [33]

$$
\mu_{e} \cong \frac{1}{2} \sin \theta_{W} \cdot e c \cdot r_{e e} \cong 9.274 \times 10^{-24} \mathrm{~J} / \text { tesla }
$$

It can be suggested that electron's magnetic moment is due to the electromagnetic interaction range. Similarly magnetic moment of proton is due to the strong interaction ending range.

$$
\mu_{p} \cong \frac{1}{2} \sin \theta_{W} \cdot e c \cdot r_{\text {se }} \cong 1.46 \times 10^{-26} \mathrm{~J} / \text { tesla }
$$

If proton and neutron are the two quantum states of the nucleon, by considering the mean strong interaction range 
$\left(\frac{r_{s s}+r_{s e}}{2}\right)$, magnetic mo ment of neutron can be fitted as

$$
\mu_{n} \cong \frac{1}{2} \sin \theta_{W} \cdot e c \cdot\left(\frac{r_{s s}+r_{s e}}{2}\right) \cong 9.82 \times 10^{-27} \mathrm{~J} / \text { tesla }
$$

\section{Basic Ideas in 'Modified' Quark Super Symmetry}

Till today there is no reason for the question: why there exists 6 individual quarks? Till today no experiment reported a free fractional charge quark. Authors humble opinion is nuclear charge (either positive or negative) constitutes 6 different flavors and each flavor holds certain mass. Charged flavor can be called as a quark. It is neither a fermion nor a boson. A fermion is a container for different charges, a charge is a container for different flavors and each flavor is a container for certain matter. If charged matter rests in a fermionic container it is a fermion and if charged matter rests in a bosonic container it is a boson. The fundamental questions to be answered are : what is a charge? why and how opposite charges attracts each other? why and how there exists a fermion? and why and how there exists a boson? Here interesting thing is that if 6 flavors are existing with 6 different masses then a single charge can have one or two or more flavors simultaneously. Since charge is a common property, mass of the multiple flavor charge seems to be the geometric mean of the mass of each flavor. If charge with flavor is called as a quark then charge with multi flavors can be called as a hybrid quark. Hybrid quark generates a multi flavor baryon. It is a property of the strong interaction space time - charge. This is just like different tastes or different smells of matter. Important consequence of this idea is thatfor generating a baryon there is no need to couple 3 fractional charge quarks[26].

1. There exist nature friendly integral charge quark fermions.

2. For every integral charge quark fermion there exists a corresponding integral charge quark boson. Quark fermion and quark boson mass ratio is close to 2.2627 .

3. There exists integral charged massive quark fermi-gluons and integral charged massive quark boso-gluons. (Fermi-gluon means massive gluons having fermion behavior and boso-gluon means massive gluons having boson behavior. Quark femi-gluon can be called as the 'quark baryon' and quark boso-gluon can be called as 'quark meson').

4. Quark fermi-gluon or quark baryon masses can be expressed as $Q_{F} c^{2} \cong 0.2314\left[M_{H f}^{2} \times Q_{f}\right]^{\frac{1}{3}} c^{2}$ and Quark boso-gluon or quark meson masses can be expressed as $Q_{M} c^{2} \cong 0.2314\left[M_{H b}^{2} \times Q_{b}\right]^{\frac{1}{3}} c^{2}$ where $Q_{f}$ and $Q_{b}$ are the rest masses of quark fermion and quark boson respectively and $M_{H f}$ and $M_{H b}$ are the Higgs charged fermion and
Higgs charged boson respectively.

5. $Q_{e f} \cong Q_{f}-Q_{b} \cong\left(1-\frac{1}{\Psi}\right) Q_{f}$ acts as the effective quark fermion. Effective quark baryon mass can be expressed as $Q_{E} c^{2} \cong 0.2314\left[M_{H f}^{2} \times Q_{e f}\right]^{\frac{1}{3}} c^{2}$. These effective quark baryons play a vital role in fitting the unstable baryon masses. Quark meson masses play a vital role in fitting the unstable meson masses.

6. Characteristic nuclear fermion is $938.272 \mathrm{MeV}$ and its corresponding nuclear boson is $\frac{938.272}{\Psi} \cong 414.67 \mathrm{MeV}$. This boson couples with the light quark bosons or light quark mesons and generates neutral ground states. Thus it is the mother of presently believed strange mesons like 493, 548, $1020 \mathrm{MeV}$ and 783, $890 \mathrm{MeV}$ etc.

7. Charged ground state baryon rest energy is $\left(Q_{E 1} Q_{E 2}\right)^{\frac{1}{2}} c^{2} \quad$ or $\left(Q_{E 1} Q_{E 2}^{2}\right)^{\frac{1}{3}} c^{2}$ or $\left(Q_{E 1} Q_{E 2} Q_{E 3}\right)^{\frac{1}{3}} c^{2}$ where $Q_{E 1}, Q_{E 2}$, and $Q_{E 3}$ represents any three effective quark baryons. Integral charge light quark bosons in one or two numbers couples with the ground or excited effective quark baryons and generates doublets and triplets. This is just like 'absorption of photons by the electron'.

8. Rest energy of nucleon is close to $\left(\frac{2 U_{F} D_{F}}{U_{F}+D_{F}}\right) c^{2} \cong 940.02 \mathrm{MeV}$ and nucleon rest energy difference is close to $\left(m_{n}-m_{y}\right) c^{2} \cong \sin ^{2} \theta_{W} \cdot\left(\frac{2 U_{f} D_{f}}{U_{f}+D_{f}}\right) c^{2} \cong 1.29623 \mathrm{MeV}$

9. Only oppositely charged quark mesons couples together to form a neutral meson. No two quark fermions couples together to form a meson. Neutral ground state meson rest energy is close to $\left(Q_{M 1}+Q_{M 2}\right) c^{2}$ where $Q_{M 1}$ and $Q_{M 2}$ represents any two quark mesons.

10. Fine rotational levels of any ground state energy $m_{x} c^{2}$ can be expressed as, if $\mathrm{n}=1,2,3 .$. , and $I=n(n+1),\left(m c^{2}\right)_{I} \cong[I]^{\frac{1}{4}} m_{x} c^{2}$ and $\left(m c^{2}\right)_{I / 2} \cong\left[\frac{I}{2}\right]^{\frac{1}{4}} m_{x} c^{2}$. Super fine rotational levels can be obtained as $\left(m c^{2}\right)_{I} \cong[I]^{\frac{1}{12}} m_{x} c^{2}$ and $\left(m c^{2}\right)_{I / 2} \cong\left[\frac{I}{2}\right]^{\frac{1}{12}} m_{x} c^{2}$.

\subsection{To Fit the Muon and Tau Rest Masses}

Using $X_{E}$ charged muon and tau masses [34] were fitted in the following way.

$$
m_{l} c^{2} \approx \frac{2}{3}\left[a_{c}^{3}+\left(n^{2} X_{E}\right)^{n} a_{a}^{3}\right]^{\frac{1}{3}}
$$

where $a_{c}$ and $a_{a}$ are the coulombic and asymmetric energy 
coefficients of the semi empirical mass formula and $n=0,1,2$. This is an approximate relation. Qualitatively this expression is connected with $\beta$ decay. Accuracy can be improved with the following relation.

$$
\text { If } \begin{aligned}
\mathrm{E}_{\mathrm{W}} \cong \frac{\mathrm{m}_{\mathrm{e}} \mathrm{c}^{2}}{\mathrm{X}_{\mathrm{E}}} \cong 1.731843735 \times 10^{-3} \mathrm{MeV} \\
m_{l} c^{2} \cong\left[X_{E}^{3}+\left(n^{2} X_{E}\right)^{n} \sqrt{N}\right]^{\frac{1}{3}} E_{W}
\end{aligned}
$$

where $n=0,1,2$. Please refer the published papers for the mystery of electro weak bosons and the Higgs boson[25,26]. Please see table-1.

Table 1. To fit the muon and tau rest masses

\begin{tabular}{|c|c|c|}
\hline $\mathrm{n}$ & $\begin{array}{c}\text { Obt. Lep. energy } \\
(\mathrm{MeV})\end{array}$ & Exp. Lep. energy (MeV) \\
\hline 0 & Defined & $0.510998910(13)$ \\
\hline 1 & 105.951 & $105.6583668(38)$ \\
\hline 2 & 1777.384 & $1776.99(29)$ \\
\hline
\end{tabular}

\subsection{To Correlate the Electron, Muon, Proton and the Charged Pion Rest Masses}

From the above table-1, if $m_{\mu} c^{2} \cong 105.95 \mathrm{MeV}$, surprisingly it is noticed that,

$$
m_{p} c^{2} \cong \frac{1}{\alpha} \cdot\left(\sqrt{m_{\mu} m_{e}}-m_{e}\right) \cong 938.29 \mathrm{MeV}
$$

Based on the proposed SUSY, it is also noticed that

$$
\left(m_{\mu} c^{2}\right)^{ \pm} \cong \frac{1}{\Psi} \cdot \sqrt{m_{\mu} m_{p}} \cong 139.34 \mathrm{MeV}
$$

These two obtained mass units can be compared with the proton and the charged pion rest masses respectively. In a unified scheme these interesting observations cannot be ignored.

\subsection{Nucleons, up \& down Quarks and the Strong Coupling Constant}

It our earlier published papers $[25,26]$ it was also defined that

$$
\frac{m_{u} c^{2}}{m_{e} c^{2}} \cong e^{X_{E} \alpha}
$$

where $m_{u}$ is the up quark rest mass and $m_{d}$ is the down quark rest mass respectively. In our earlier papers, suggested up quark mass is $4.4 \mathrm{MeV}$ and down quark mass is 9.476 $\mathrm{MeV}$. With these magnitudes it is noticed that,

$$
\left(m_{n}-m_{p}\right) c^{2} \cong \ln \left(\frac{\sqrt{m_{u} m_{d}}}{m_{e}}\right) m_{e} c^{2}
$$

Here lhs $=1.2933 \mathrm{MeV}$ and rhs $=1.2963 \mathrm{MeV}$. It is also noticed that

$$
\left(\frac{\sqrt{m_{u} m_{d}}}{m_{e}}\right) \cong \frac{1}{2} \sqrt{\frac{G M_{X}^{2}}{\hbar c}} \cong 12.60271
$$

With reference to the strong coupling constant $\alpha_{s}$ - it is also noticed that[19],

$$
\begin{gathered}
\left(\frac{1}{\alpha}+\frac{1}{\alpha_{s}}\right) \sqrt{m_{u} m_{d}} c^{2} \cong 940 \mathrm{MeV} \\
\frac{\sqrt{m_{u} m_{d}} c^{2}}{\left(m_{n}-m_{p}\right) c^{2}} \cong \ln \left(\frac{1}{\alpha}+\frac{1}{\alpha_{s}}\right)
\end{gathered}
$$

\subsection{To Fit the Strong Coupling Constant}

The strong coupling constant $\alpha_{s}$ is a fundamental parameter of the Standard Model. It plays a more central role in the QCD analysis of parton densities in the moment space. Considering perturbative QCD calculations from threshold corrections, its recent obtained value[35] at is $\mathrm{N}^{3} \mathrm{LO}$ $\alpha_{s} \cong 0.1139 \pm 0.0020$. At lower side $\alpha_{s} \cong 0.1139-0.002=0.1119$ and at higher side $\alpha_{s} \cong 0.1139+0.002=0.1159$. It can be fitted or defined in the following way.

$$
\begin{aligned}
X_{S} \cong \frac{1}{\alpha_{s}} \cong \ln \sqrt{\frac{1}{\alpha}\left(\frac{r_{e e}}{r_{s e}}\right)^{2}} \cong \ln \sqrt{\frac{1}{\alpha}\left(\frac{G M_{X}^{2}}{\hbar c}\right)^{2}} \\
\cong 8.914239916 \\
\alpha_{s} \cong \frac{1}{X_{S}} \cong \frac{1}{\ln \left(X_{E}^{2} \sqrt{\alpha}\right)} \cong 0.112180063
\end{aligned}
$$

This proposed value numerically can be compared with the current estimates of the $\alpha_{s}$. Here note that the proposed definition considers the electromagnetic and strong interaction ranges in a unified manner. This proposal can be given a chance[25]. With this magnitude it is noticed that

$$
\begin{aligned}
m_{n} c^{2} & \cong\left(\frac{1}{\alpha}+\frac{1}{\alpha_{s}}\right) \sqrt{m_{u} m_{d}} c^{2}-\frac{m_{u}}{m_{d}}\left(\frac{2 m_{u} m_{d}}{m_{u}+m_{d}}\right) c^{2} \\
& \cong 939.6 \mathrm{MeV} \\
m_{p} c^{2} & \cong\left(\frac{1}{\alpha}+\frac{1}{\alpha_{s}}\right) \sqrt{m_{u} m_{d}} c^{2}-\sqrt{\frac{m_{u}}{m_{d}}}\left(\frac{2 m_{u} m_{d}}{m_{u}+m_{d}}\right) c^{2} \\
& \cong 938.30 \mathrm{MeV}
\end{aligned}
$$

where $\left(\frac{1}{\alpha}+\frac{1}{\alpha_{s}}\right) \sqrt{m_{u} m_{d}} c^{2} \cong 942.393 \mathrm{MeV}$.

\section{Integral Charge Quark Fermions and Their SUSY Bosons}

In the previous papers[25] authors suggested that up, strange and bottom quarks are in geometric series. Similarly down, charm and top quarks are in another geometric series. Obtained quark fermion masses can be compared with the current estimates. Up and down fermion masses can be given as

$$
U_{f} c^{2} \cong e^{\alpha X_{E}} \times m_{e} c^{2} \cong 4.4 \mathrm{MeV}
$$




$$
D_{f} c^{2} \cong \alpha X_{E} \times U_{f} c^{2} \cong 9.4755 \mathrm{MeV}
$$

It is very interesting to note that

$$
\frac{\text { Down fermion mass }}{\text { Up fermion mass }} \cong \frac{D_{f}}{U_{f}} \cong \alpha X_{E} \cong \frac{1}{\sin \theta_{W}}
$$

In this way $\sin \theta_{W}$ can be related with up and down quark mass ratio. Proposed USB geo metric ratio is

$$
g_{U} \cong\left[\alpha X_{E} \frac{\alpha X_{E}+1}{\alpha X_{E}-1}\right]^{2} \cong 34.66294
$$

If DCT series is the second generation series, its geometric ratio is

$$
\begin{gathered}
g_{D} \cong\left[2 \alpha X_{E} \frac{\alpha X_{E}+1}{\alpha X_{E}-1}\right]^{2} \cong 138.651754 \\
\frac{g_{D}}{g_{U}} \cong \frac{\text { DCT geometric ratio }}{\text { USB geometric ratio }} \cong 4 .
\end{gathered}
$$

Quark boson mass $=Q_{b} \cong \frac{\text { Quark fermion mass }}{\Psi} \cong \frac{Q_{f}}{\Psi}$

Table 2. Fitting of quark fermion and quark boson masses

\begin{tabular}{|c|c|c|}
\hline Quark & $Q_{f} c^{2}$ in MeV & $Q_{b} c^{2}$ in MeV \\
\hline Up & 4.401 & 1.945 \\
\hline Down & 9.4755 & 4.188 \\
\hline Strange & 152.5427 & 67.416 \\
\hline Charm & 1313.796 & 580.63 \\
\hline Bottom & 5287.579 & 2336.839 \\
\hline Top & 182160.18 & 80505.46 \\
\hline
\end{tabular}

Please see table-2 for the obtained quark 'fermion' and 'boson' masses. The observed baryon and meson charge-mass spectrum can be generated from these mass units. Strange quark boson pair generates the neutral pion of rest energy $134.83 \mathrm{MeV}$. Obtained top quark boson rest energy is $80505 \mathrm{MeV}$ and is very close to the observed $\mathrm{W}$ boson rest energy $80.450 \pm 0.058 \mathrm{GeV}$ and $80.392 \pm 0.039$

GeV. Please refer M. Yao et al[34] recommended PDG data. Really this is a great coincidence and support for the proposed new idea of "fermion-boson" unification scheme. This strongly supports super symmetry with small modifications.

\subsection{Beta Decay, Higg's Charged Fermi on and its B os on}

It is well established that in Beta decay electron is instantaneously created from neutron and this nuclear weak force is mediated by $W$ and $Z$ bosons. If $W$ boson is really the SUSY partner of top quark then the role of $\mathrm{W}$ boson in weak decay seems to be nothing. Its role is taken up by the newly proposed Higgs charged boson of rest energy close to 45.6 GeV. Mass of $\Psi M_{H b}$ or $M_{H f}$ can be expressed with the following relations.

$$
\begin{array}{r}
M_{H f} c^{2} \cong \frac{1}{2} \cdot\left(\frac{G M_{X}^{2}}{\hbar c}\right)^{2} \cdot w_{e} z \exists z 5.417 \mathrm{MeV} \\
M_{H b} c^{2} \cong \frac{M_{H f} c^{2}}{\Psi} \cong \frac{1}{2} \cdot\left(\frac{G M_{X}^{2}}{\hbar c}\right)^{2} \cdot \frac{m_{f} \xi^{2}}{\Psi} 76 \fallingdotseq 467 \mathrm{MeV}
\end{array}
$$

\subsection{Rest Energy of the Neutral $Z$ Boson}

From above estimation, neutral $Z$ boson rest energy can be given as

$$
\begin{gathered}
m_{Z} c^{2} \cong\left(M_{H b} c^{2}\right)^{ \pm}+\left(M_{H b} c^{2}\right)^{\mp} \cong 2 M_{H b} c^{2} \cong 91152.293 \mathrm{MeV}(5 \\
m_{Z} c^{2} \cong\left(\frac{G M_{X}^{2}}{\hbar^{2}}\right)^{2} \cdot \frac{m_{e} c^{2}}{\Psi} \cong 91152.293 \mathrm{MeV}
\end{gathered}
$$

This obtained value can be compared with the experimental rest energy of $Z$ boson $=91187.621 \mathrm{MeV}$. Please refer M. Yao et al reco mmended PDG data[34].

\subsection{Recently Discovered Boson of Rest Energy $126 \mathrm{GeV}$}

Close to the predicted rest energy of Higgs boson, recently a new boson of rest energy 124 to $160 \mathrm{GeV}$ was reported. Surprising thing is that its existence is not matching with the current theoretical predictions. In this critical situation, with the help of strong nuclear gravity and modified super symmetry concepts, authors made an attempt to understand the origin of this new boson[26]. In our previous paper[25] it was suggested that: $W$ boson is the super symmetric boson of the top quark fermion and the charged Higgs boson pair generates the neutralized $Z$ boson. It is noticed that Higgs charged boson and top quark boson couples together to form a new neutral boson of rest energy $126.0 \mathrm{GeV}$. This is a very interesting observation. Like $Z$ boson it can decay into 2 charged particles.

$$
\left(M_{H b} c^{2}\right)^{ \pm}+\left(m_{W} c^{2}\right)^{\mp} \cong 126.0 \mathrm{GeV} .
$$

Table 3. Fitting of quark baryon and quark effective baryon rest energies

\begin{tabular}{|c|c|c|c|c|}
\hline \multirow{2}{*}{ Quark } & $Q_{f} c^{2}$ in MeV & $Q_{F} c^{2}$ in $\mathrm{MeV}$ & $Q_{e f} c^{2}$ in $\mathrm{MeV}$ & $Q_{E} c^{2}$ in $\mathrm{MeV}$ \\
\hline Up & 4.401 & 834.04 & 2.456 & 686.66 \\
\hline Down & 9.4755 & 1076.97 & 5.2878 & 886.67 \\
\hline Strange & 152.5427 & 2719.35 & 85.127 & 2238.84 \\
\hline Charm & 1313.796 & 5574.13 & 733.165 & 4589.18 \\
\hline Bottom & 5287.579 & 8866.53 & 2950.74 & 7299.81 \\
\hline Top & 182160.18 & 28850.43 & 101654.72 & 23752.56 \\
\hline
\end{tabular}




\subsection{Quark Baryon and Quark Meson Masses with S USY Higg's Charged Particle}

In our earlier published paper it was assumed that[25], if $Q_{F}$ is the quark baryon rest mass

$$
Q_{F} c^{2} \cong\left[M_{G f}^{2} \cdot Q_{f}\right]^{\frac{1}{3}} c^{2}
$$

If $Q_{E}$ is the quark effective baryon rest mass,

$$
Q_{E} c^{2} \cong\left[M_{G f}^{2} \cdot Q_{e f}\right]^{\frac{1}{3}} c^{2}
$$

If $Q_{M}$ is the quark meson rest mass,

$$
Q_{M} c^{2} \cong\left[M_{G b}^{2} \cdot Q_{b}\right]^{\frac{1}{3}} c^{2}
$$

where $M_{G f} c^{2} \cong 11460 \mathrm{MeV}$ and its bosonic form $M_{G b} c^{2} \cong \frac{M_{G f} c^{2}}{\Psi} \cong 5066 \mathrm{MeV}$.With reference to the newly proposed Higgs charged fermion and boson, above relations can be expressed as

$$
\begin{aligned}
& Q_{F} c^{2} \cong x\left[M_{H f}^{2} \cdot Q_{f}\right]^{\frac{1}{3}} c^{2} \\
& Q_{E} c^{2} \cong x\left[M_{H f}^{2} \cdot Q_{e f}\right]^{\frac{1}{3}} c^{2} \\
& Q_{M} c^{2} \cong x\left[M_{H b}^{2} \cdot Q_{b}\right]^{\frac{1}{3}} c^{2}
\end{aligned}
$$

$$
\text { where } \mathrm{x} \cong \frac{1}{2 \alpha\left(\mathrm{X}_{\mathrm{E}}+1\right)} \cong 0.23143232
$$

Please see table- 3 for the quark baryon rest energies and see table-4 for the quark meson rest energies.

Table 4. Fitting of quark boson and quark meson rest energies

\begin{tabular}{|c|c|c|}
\hline Quark & $Q_{b} c^{2}$ in $\mathrm{MeV}$ & $Q_{M} c^{2}$ in $\mathrm{MeV}$ \\
\hline Up & 1.945 & 368.6 \\
\hline Down & 4.188 & 475.98 \\
\hline Strange & 67.416 & 1201.81 \\
\hline Charm & 580.63 & 2463.48 \\
\hline Bottom & 2336.839 & 3918.55 \\
\hline Top & 80505.46 & 12750.41 \\
\hline
\end{tabular}

\subsection{Rest Energy of the Nucle on}

From table-3 it is noticed that, nucleon mass is very close to the harmonic mean of the up baryon and down baryon masses.

$$
\frac{2\left(U_{F} c^{2}\right)\left(D_{F} c^{2}\right)}{\left(U_{F}+D_{F}\right) c^{2}} \cong 940.06 \mathrm{MeV}
$$

where $U_{F} c^{2} \cong 834.04 \mathrm{MeV}$ and $D_{F} c^{2} \cong 1076.97 \mathrm{MeV}$. It is also noticed that,

$$
\left(m_{n}-m_{p}\right) c^{2} \cong \sin ^{2} \theta_{W}\left[\frac{2\left(U_{f} c^{2}\right)\left(D_{f} c^{2}\right)}{\left(U_{f}+D_{f}\right) c^{2}}\right] \cong 1.2964 \mathrm{MeV}
$$

where $m_{p}$ and $m_{n}$ are the rest masses of proton and neutron respectively.

\section{To Fit the Semi Empirical Mass Formula Energy Coefficients}

The semi-empirical mass formula (SEMF) is used to approximate the mass and various other properties of an atomic nucleus[36,37]. As the name suggests, it is based partly on theory and partly on empirical measurements. The theory is based on the liquid drop model proposed by George Gamow and was first formulated in 1935 by German physicist Carl Friedrich von Weizsäcker. Based on the 'least squares fit', volume energy coefficient is $a_{v}=15.78 \mathrm{MeV}$, surface energy coefficient is $a_{s}=18.34 \mathrm{MeV}$, coulombic energy coefficient is $a_{c}=0.71 \mathrm{MeV}$, asymmetric energy coefficient is $a_{a}=23.21 \mathrm{MeV}$ and pairing energy coefficient is $a_{p}=12 \mathrm{MeV}$. The semi empirical mass formula is

$$
B E \cong A a_{v}-A^{\frac{2}{3}} a_{s}-\frac{Z(Z-1)}{A^{\frac{1}{3}}} a_{c}-\frac{(A-2 Z)^{2}}{A} a_{a} \pm \frac{1}{\sqrt{A}} a_{p}(63)
$$

In a unified approach it is noticed that, the energy coefficients are having strong inter-relation with the proton rest mass and the 'mole electron mass'. The interesting observations can be expressed in the following way.

\subsection{The Coulombic Energy Coefficient}

It can be defined as[38],

$$
a_{c} \cong \alpha \cdot \alpha_{s} \cdot m_{p} c^{2} \cong 0.7681 \mathrm{MeV}
$$

Ratio of the coulombic energy coefficient and the proton rest energy is close to the product of the fine structure ratio and the strong coupling constant.

\subsection{The Surface and Volume Energy Coefficients}

Surface energy coefficient can be defined as

$$
a_{s} \cong \sqrt{\frac{G M_{X}^{2}}{\hbar c}} 3 a_{c} \cong 9.36 \mathrm{MeV}
$$

Volu me energy coefficient can be defined as

$$
\begin{aligned}
& a_{v} \cong \sqrt{\frac{G M_{X}^{2}}{\sqrt{2} \hbar c}} 2 a_{c} \cong 6.28 \mathrm{MeV} \\
& \text { Thus, } \frac{\mathrm{a}_{\mathrm{s}}}{\mathrm{a}_{\mathrm{v}}} \cong 2^{\frac{1}{4}}
\end{aligned}
$$

\subsection{The Asymmetry and Pairing Energy Coefficients}

Asymmetry energy coefficient can be defined as

$$
a_{a} \cong \frac{2}{3}\left(a_{v}+a_{s}\right) \cong 23.76 \mathrm{MeV}
$$

Pairing energy coefficient is close to

$$
a_{p} \cong \frac{1}{3}\left(a_{v}+a_{s}\right) \cong 11.88 \mathrm{MeV}
$$


Thus, $\mathrm{a}_{\mathrm{v}}+\mathrm{a}_{\mathrm{s}} \cong \mathrm{a}_{\mathrm{a}}+\mathrm{a}_{\mathrm{p}} \cong 35.64 \mathrm{MeV}$

Table 5. SEMF binding energy with the proposed energy coefficients

\begin{tabular}{|c|c|c|c|}
\hline$Z$ & $A$ & $(B E)_{c a l}$ in $\mathrm{MeV}$ & $(B E)_{\text {mes }}$ in $\mathrm{MeV}$ \\
\hline 26 & 56 & 492.60 & 492.254 \\
\hline 28 & 62 & 547.08 & 545.259 \\
\hline 34 & 84 & 728.29 & 727.341 \\
\hline 50 & 118 & 1007.46 & 1004.950 \\
\hline 60 & 142 & 1183.64 & 1185.145 \\
\hline 79 & 197 & 1554.82 & 1559.40 \\
\hline 82 & 208 & 1625.22 & 1636.44 \\
\hline 92 & 238 & 1803.12 & 1801.693 \\
\hline
\end{tabular}

In table-5 considering the magic numbers, within the range of $(Z=26 ; A=56)$ to $(Z=92 ; A=238)$ nuclear binding energy is calculated and compared with the measured binding energy[39]. Column-3 represents the calculated binding energy and column-4 represents the measured binding energy. If this procedure is found to be true and valid then with a suitable fitting procedure qualitatively and quantitatively magnitudes of the proposed SEMF binding energy coeffic ients can be refined.

\subsection{Proton-Nucleon Stability}

It is noticed that

$$
\frac{A_{s}}{2 Z} \cong 1+2 Z\left(\frac{a_{c}}{a_{s}}\right)^{2} \cong 1+2 Z\left(\frac{\hbar c}{G M_{X}^{2}}\right)
$$

where $A_{s}$ is the stable mass number of $Z$. This is a direct relation. Assuming the proton number $Z$, in general, for all atoms, lower stability can be fitted directly with the following relation[33].

$$
A_{s} \cong 2 Z\left[1+2 Z\left(\frac{a_{c}}{a_{s}}\right)^{2}\right] \cong 2 Z+Z^{2} * 0.0063
$$

if $\quad Z=21, \quad A_{s} \cong 44.78 ; \quad$ if $\quad Z=29, \quad A_{s} \cong 63.29 ; \quad$ if $Z=47, \quad A_{s} \cong 107.91 ; \quad$ if $\quad Z=53, \quad A_{s} \cong 123.68 ; \quad$ if $Z=60, \quad A_{s} \cong 142.66 ; \quad$ if $\quad Z=79, \quad A_{s} \cong 197.29 ; \quad$ if $Z=83, \quad A_{s} \cong 209.37 ;$ if $Z=92, A_{s} \cong 237.29$;

Stable super heavy elements can be predicted with this relation. In between $Z=30$ to $Z=60$ obtained $A_{s}$ is lower compared to the actual $A_{s}$. It is noticed that, upper stability in light and medium atoms upto $Z \approx 56$ can be fitted with the following relation.

$$
\begin{aligned}
A_{s} & \cong 2 Z\left[1+2 Z\left(\left(\frac{a_{c}}{a_{s}}\right)^{2}+\left(\frac{a_{c}}{a_{a}+a_{p}}\right)^{2}\right)\right] \\
& \cong 2 Z+Z^{2} * 0.0082
\end{aligned}
$$

From this relation for $Z=56$, obtained upper $A_{s} \cong 137.7$. Note that, for $Z=56$, actual stable
$A_{s} \cong 137 \cong \frac{1}{\alpha}$ where $\alpha$ is the fine structure ratio. This seems to be a nice and interesting coincidence. In between 0.0063 and 0.0082 , for light and medium atoms up to $Z \approx 56$ or $A_{s} \approx 137$, mean stability can be fitted with the following relation.

$$
A_{s} \cong 2 Z+Z^{2} * 0.0072
$$

Surprisingly it is noticed that, in this relation, $0.0072 \approx \alpha \cong 0.0073$. Thus up to $Z \cong 56$ or $A_{s} \approx 137$, mean stability can be expressed as

$$
A_{s} \approx 2 Z+\left(Z^{2} \alpha\right)
$$

\subsection{Nucle ar Binding Energy with 2 Ter ms and Only One Energy Constant}

Nuclear binding energy can be fitted with 2 terms or 4 factors with $a_{c} \cong 0.7681 \mathrm{MeV}$ as the single energy constant[33,40]. First term can be expressed as

$$
T_{1} \cong(f)(A+1) \ln \left[(A+1) X_{S}\right] a_{c}
$$

where $f \cong 1+\frac{2 Z}{A_{S}} \leq 2.0$ and $X_{S} \cong 8.91424$ is the strong coupling constant.

Second term can be expressed as

$$
T_{2} \cong\left[\frac{A^{2}+\left(f \cdot Z^{2}\right)}{X_{S}^{2}}\right] a_{c}
$$

Close to the stable mass number $A_{S}$,

$$
\text { B. } E=T_{1}-T_{2}
$$

Please see the following data.

$Z=2 \quad \& \quad A=4, B . E \cong 28.93 \mathrm{MeV}$;

$Z=10 \quad \& \quad A=20, B . E \cong 160.44 \mathrm{MeV}$;

$Z=26 \& A=56, B . E \cong 482.06 \mathrm{MeV}$;

$Z=50 \& A=118, B . E \cong 1007.35 \mathrm{MeV}$;

$Z=79 \& A=197, B . E \cong 1563.72 \mathrm{MeV}$;

$Z=82 \quad \& \quad A=208, B . E \cong 1634.76 \mathrm{MeV}$;

$Z=92 \quad \& \quad A=238, B . E \cong 1805.15 \mathrm{MeV}$.

Above 2 terms can be put into 4 factors as

$$
B . E \cong\left[2-\frac{A}{2 Z}\right](f)(A+1) \ln \left[(A+1) X_{S}\right] a_{c}
$$

With this relation,

$$
\begin{aligned}
& Z=2 \quad \& \quad A=4, B . E \cong 29.07 \mathrm{MeV} ; \\
& Z=10 \quad \& \quad A=20, B . E \cong 160.98 \mathrm{MeV} ; \\
& Z=26 \quad \& \quad A=56, B . E \cong 484.56 \mathrm{MeV} ; \\
& Z=50 \quad \& \quad A=118, B . E \cong 973.32 \mathrm{MeV} ; \\
& Z=79 \quad \& \quad A=197, B . E \cong 1542.1 \mathrm{MeV} ; \\
& Z=82 \quad \& \quad A=208, B . E \cong 1587.52 \mathrm{MeV} ; \\
& Z=92 \quad \& \quad A=238, B . E \cong 1764.8 \mathrm{MeV} ;
\end{aligned}
$$

These relations can be considered for further research and analysis positively. 


\section{The Characteristic Nuclear Radii in Cosmology}

Please recall that, the characteristic cosmic Hubble mass is $M_{0} \cong \frac{c^{3}}{2 G H_{0}} \cong 8.95 \times 10^{52} \mathrm{Kg}$ and the electromagnetic mass of the proposed $M_{X}$ is $M_{C} \cong \sqrt{\frac{e^{2}}{4 \pi \varepsilon_{0} G}} \cong 1.859210775 \times 10^{-9} \mathrm{Kg}$.

\subsection{The Characteristic Nuclear Charge Radius}

If $H_{0} \cong 69.54 \mathrm{Km} / \mathrm{sec} / \mathrm{Mpc}, R_{S}$ is the characteristic radius of nucleus, it is noticed that,

$$
R_{S} \cong\left(\frac{m_{p}}{M_{X}}\right)^{2} \frac{c}{H_{0}} \cong 1.2368 \times 10^{-15} \mathrm{~m}
$$

where $m_{p}$ is the proton rest mass. This can be compared with the characteristic radius of the nucleus and the strong interaction range[29].

\subsection{Scattering Distance between Electron and the Nucleus}

If $R_{S} \cong 1.21$ to $1.22 \mathrm{fm}$ is the minimum scattering distance between electron and the nucleus, it is noticed that,

$$
\begin{aligned}
R_{S} & \cong\left(\frac{\hbar c}{G M_{X}^{2}}\right) \cdot\left(\frac{\hbar c}{G m_{e}^{2}}\right) \cdot \frac{2 G m_{e}}{c^{2}} \\
& \cong 1.21565 \times 10^{-15} \mathrm{~m}
\end{aligned}
$$

Here $M_{X}$ is the molar electron mass. Here it is very interesting to consider the role of the Schwarzschild radius of the 'electron mass'. Thus the two macroscopic physical constants $N$ and $G$ can be expressed in the following way.

$$
\begin{gathered}
N \cong \sqrt{\frac{2 \hbar^{2}}{G m_{e}^{3} R_{S}}} \\
G \cong \frac{2 \hbar^{2}}{\left(M_{X}\right)^{2} m_{e} R_{S}}
\end{gathered}
$$

In this way, either the Avogadro number or the gravitational constant can be obtained. Combining the relations (80) and (81) and if $H_{0} \cong 69.54 \mathrm{Km} / \mathrm{sec} / \mathrm{Mpc}$, it is noticed that,

$$
\frac{\hbar c}{G m_{p} \sqrt{M_{0} m_{e}}} \cong 0.991415
$$

Surprisingly this ratio is close to unity! How to interpret this ratio? From this relation it can be suggested that, along with the cosmic variable, $H_{0}$, in the atomic and nuclear physics, there exists one variable. In the physics history, it was suggested that, gravitational constant and the speed of light were cosmic variables. In our published paper[41] and accepted paper[42] it was assumed that, the reduced Planck's constant, the Bohr radius, the fine structure ratio were cosmic variables. In our another accepted paper[43] it was assumed that, proton mass and the proton radius were cosmic variables. Any how this is a very sensitive case and has to be discussed in depth. But it is clear that, on the cosmological time scale, there exists one variable physical quantity in the presently believed atomic and nuclear physical constants. 'Rate of change' in its magnitude may be a measure of the present cosmic acceleration. Thus independent of the cosmic red shift and CMBR observations, from the atomic and nuclear physics, cosmic acceleration can be verified. Based on the above coincidence, magnitude of the present Hubble's constant can be expressed as

$$
H_{0} \cong \frac{G m_{p}^{2} m_{e} c}{2 \hbar^{2}} \cong 70.75 \mathrm{Km} / \mathrm{sec} / \mathrm{Mpc}
$$

\subsection{To Fit the Radius of Proton}

Let $R_{p}$ be the radius of proton. It is noticed that,

$$
R_{y} \cong \frac{M_{X}}{m_{y}} \cdot \frac{2 G M_{C}}{c^{2}} \cong 9.0566 \times 10^{-16} \mathrm{~m}
$$

This obtained magnitude can be compared with the rms charge radius of the proton[30]. W ith different experimental methods its magnitude varies from $0.84184(67) \mathrm{fm}$ to $0.895(18) \mathrm{fm}$. Here also it is very interesting to consider the role of the Schwarzschild radius of $M_{C}$. This type of coincidence cannot be ignored in the unification scheme. Here the strange observation is: the ratio $\frac{M_{X}}{m_{p}}$. Please note that mass nature in both of the cases is the assumed 'gravitational mass' only. But the very strange observation is $\frac{2 G M_{C}}{c^{2}}$. Here in this expression, $M_{C}$ is playing a key role instead of $M_{X}$. But $M_{C}$ is the assumed electromagnetic mass of $M_{X}$. If this logic is having any sense, then similar to $M_{C}$, 'electro magnetic mass of the proton' must play a strong role in nuclear physics. In this direction, in the following subsection, an attempt is made.

\subsection{Strong Interaction Range in Cos mology}

Considering the above coincidences it can be suggested that, there exists a strong connection in between modern cosmology and the nucleus. It is noticed that,

$$
R_{S} \cong \frac{2 G \sqrt{M_{0}\left(m_{p} / X_{E}\right)}}{c^{2}} \cong 1.0493 \times 10^{-15} \mathrm{~m}
$$

where $H_{0} \cong 70.75 \mathrm{Km} / \mathrm{sec} / \mathrm{Mpc}$ and $M_{0} \cong 8.80434 \times 10^{52}$ $\mathrm{Kg}$. Here $R_{S}$ represents the represents the Schwarzschild radius of $\sqrt{M_{0}\left(m_{p} / X_{E}\right)}$. How to understand this radius! Here the very peculiar and careful observation is

$$
\begin{gathered}
R_{S} \cong \frac{2 G \sqrt{M_{0}\left(m_{p} / X_{E}\right)}}{c^{2}} \cong \sqrt{\left(\frac{2 G M_{0}}{c^{2}}\right)\left(\frac{2 G\left(m_{p} / X_{E}\right)}{c^{2}}\right)} \\
\cong 1.0493 \times 10^{-15} \mathrm{~m}
\end{gathered}
$$


In this relation, $\frac{2 G M_{0}}{c^{2}}$ is the Schwarzschild radius of the Hubble mass! It means, from unification point of view[10,11], if the above relation (88) receives any significance, then it can be suggested that, in the flat universe, for any observer - cosmic observations and events seems to be confined to the Hubble volu me[44]. So me scientists may say: this is a play with numbers. Some scientists may say: it seems to be a fun. Some scientists may say: it is very interesting. Some scientists say: nobody understands Mach's principle this way. Here, the fundamental question to be answered is - if the atom (and therefore all material rulers) expands, in what relation should the cosmic expansion then be measured? Answer is very simple. If the universe is really accelerating, based on the galactic red shift, for the observerthe receding and accelerating galaxy must show a continuous increase in its red shift[44]. There is no such evidence. If we do not yet know whether the universe is spatially closed or open, then the idea of Hubble mass can be used as a tool in cosmology and unification. Considering the particle and event horizon concepts, where ever we go in the flat universe, for the observer, Hubble volume is the only observable/workable volume. Hence where ever we go in the universe, Hubble mass plays the role. It is very close to the Mach's idea of distance cosmic back ground. It seems to be a quantitative description to the Mach's principle. Anyhow whatever may be their physical meaning, it is sure that these relations will help in understanding the characteristic properties of strong interaction, unification, cosmic acceleration and Mach's principle.

\section{Cosmic Critical Density, Matter Density and Thermal Energy Density}

It is noticed that, there exists a very simple relation in between the cosmic critical density, matter density and the thermal energy density. It can be expressed in the following way. At any time $t$,

$$
\left(\frac{\rho_{c}}{\rho_{m}}\right)_{t} \cong\left(\frac{\rho_{m}}{\rho_{T}}\right)_{t} \cong 1+\ln \left(\frac{M_{t}}{M_{C}}\right)
$$

where $\rho_{c} \cong M_{t}\left[\frac{4 \pi}{3}\left(\frac{c}{H_{t}}\right)^{3}\right]^{-1} \cong \frac{3 H_{t}^{2}}{8 \pi G}, \quad \rho_{m}$ is the matter density and $\rho_{T}$ is the thermal energy density expressed in gram $/ \mathrm{cm}^{3}$ or $\mathrm{Kg} / \mathrm{m}^{3}$. Considering the Planck - Coulo mb scale, at the beginning if $M_{t} \cong M_{C}$

$$
\begin{gathered}
\left(\frac{\rho_{c}}{\rho_{m}}\right)_{C} \cong\left(\frac{\rho_{m}}{\rho_{T}}\right)_{C} \cong 1 \\
\left(\rho_{c}\right)_{C} \cong\left(\rho_{m}\right)_{C} \cong\left(\rho_{T}\right)_{C}
\end{gathered}
$$

Thus at any time $t$,

$$
\rho_{m} \cong \sqrt{\rho_{c} \cdot \rho_{T}}
$$

$$
\begin{gathered}
\rho_{m} \cong\left[1+\ln \left(\frac{M_{t}}{M_{C}}\right)\right]^{-1} \rho_{c} \\
\rho_{T} \cong\left[1+\ln \left(\frac{M_{t}}{M_{C}}\right)\right]^{-2} \rho_{c} \cong\left[1+\ln \left(\frac{M_{t}}{M_{C}}\right)\right]^{-1} \rho_{m}
\end{gathered}
$$

In this way, observed matter density and the thermal energy density can be studied in a unified manner. The observed CMB anisotropy can be related with the inter galactic matter density fluctuations.

\subsection{Present Matter Density of the Uni verse}

At present if $H_{0} \cong 70.75 \mathrm{Km} / \mathrm{sec} / \mathrm{Mpc}$,

$$
\left(\rho_{m}\right)_{0} \cong\left[1+\ln \left(\frac{M_{0}}{M_{C}}\right)\right]^{-1}\left(\rho_{c}\right)_{0}
$$

$\cong 6.573 \times 10^{-32} \mathrm{gram} / \mathrm{cm}^{3}$

where $\left(\rho_{c}\right)_{0} \cong 9.4 \times 10^{-30} \mathrm{gram} / \mathrm{cm}^{3}$ and $\left[1+\ln \left(\frac{M_{0}}{M_{C}}\right)\right] \cong 143.013$. Based on the average mass-to-light ratio for any galaxy[6]

$$
\left(\rho_{m}\right)_{0} \cong 1.5 \times 10^{-32} \eta h_{0} \text { gram } / \mathrm{cm}^{3}
$$

where for any galaxy, $\left\langle\frac{M_{G}}{L_{G}}\right\rangle \cong \eta\left(\frac{M_{\odot}}{L_{\odot}}\right)$ and the number $h_{0} \cong \frac{H_{0}}{100 \mathrm{Km} / \mathrm{sec} / \mathrm{Mpc}} \cong \frac{70.75}{100} \cong 0.7075$.

Note that elliptical ga laxies probably comprise about $60 \%$ of the galaxies in the universe and spiral galaxies thought to make up about $20 \%$ percent of the galaxies in the universe. Almost $80 \%$ of the galaxies are in the form of elliptical and spiral galaxies. For spiral galaxies, $\eta h_{0}^{-1} \cong 9 \pm 1$ and for elliptical galaxies, $\eta h_{0}^{-1} \cong 10 \pm 2$. For our galaxy inner part, $\eta h_{0}^{-1} \cong 6 \pm 2$. Thus the average $\eta h_{0}^{-1}$ is very close to 8 to 9 and its corresponding matter density is close to ( 6.0 to 6.76$) \times 10^{-32} \mathrm{gram} / \mathrm{cm}^{3}$ and can be compared with the above proposed magnitude of $6.573 \times 10^{-32} \mathrm{gram} / \mathrm{cm}^{3}$.

\subsection{Present ther mal Energy Density of the Uni verse}

At present if $H_{0} \cong 70.75 \mathrm{Km} / \mathrm{sec} / \mathrm{Mpc}$,

$$
\left(\rho_{T}\right)_{0} \cong\left[1+\ln \left(\frac{M_{0}}{M_{C}}\right)\right]^{-2}\left(\rho_{c}\right)_{0} \cong 4.6 \times 10^{-34} \mathrm{gram} / \mathrm{cm}^{3}(97)
$$

and thus

$$
\left(\rho_{T} c^{2}\right)_{0} \cong\left[1+\ln \left(\frac{M_{0}}{M_{C}}\right)\right]^{-2}\left(\rho_{c} c^{2}\right)_{0} \cong 4.131 \times 10^{-14} \mathrm{~J} / \mathrm{m}^{3}(98)
$$

At present if

$$
\left(\rho_{T} c^{2}\right)_{0} \cong a T_{0}^{4}
$$


where $a \cong 7.56576 \times 10^{-16} \mathrm{~J} / \mathrm{m}^{3} \mathrm{~K}^{4}$ is the radiation energy density constant, then the obtained temperature is, $T_{0} \cong 2.718{ }^{0}$ Kelvin. This is accurately fitting with the observed CMBR temperature[22], $T_{0} \cong 2.725{ }^{0}$ Kelvin.

Thus in this way, the present value of the Hubble's constant and the present CMBR temperature can be co-related with the following trial-error relation.

$$
\left[1+\ln \left(\frac{c^{3}}{2 G H_{0} M_{C}}\right)\right]^{-1} H_{0} \cong \sqrt{\frac{8 \pi G a T_{0}^{4}}{3 c^{2}}}
$$

\section{Present Cosmic Age}

Cosmic age can be assumed as

$$
t \cong\left(\frac{\rho_{c} c^{2}}{\rho_{T} c^{2}}\right)_{t}\left(\frac{1}{H_{t}}\right) \cong\left[1+\ln \left(\frac{c^{3}}{2 G H_{t} M_{C}}\right)\right]^{2}\left(\frac{1}{H_{t}}\right)
$$

Here note that, cosmic age is directly proportional to the ratio of critical energy density and the thermal energy density. In this way, this proposed method differs from the current or standard model of cosmology by the ratio $\left(\frac{\rho_{c} c^{2}}{\rho_{T} c^{2}}\right)_{0}$. Thus at any time $t$,

$$
t \cdot H_{t} \cong\left(\frac{\rho_{C} c^{2}}{\rho_{T} c^{2}}\right)_{t} \cong\left[1+\ln \left(\frac{c^{3}}{2 G H_{t} M_{C}}\right)\right]^{2}
$$

At present if $H_{0} \cong 70.75 \mathrm{Km} / \mathrm{sec} / \mathrm{Mpc}$, present cosmic age can be expressed as

$$
t_{0} \cong\left[1+\ln \left(\frac{c^{3}}{2 G H_{0} M_{C}}\right)\right]^{2}\left(\frac{1}{H_{0}}\right)
$$

i.e The present cosmic age is $8.92 \times 10^{21} \mathrm{sec} \cong 282.7$ trillion years. With this large time - smooth cosmic expansion, cosmic isotropy, super novae dimming and magnetic monopole vanishing etc can be understood. In Indian vedic cosmology, total age of the universe is 311 trillion years $[43,45]$. This is a striking and surprising coincidence. It can be suggested that, modern cosmo logy and Indian vedic cosmology can be studied in a unified manner.

\section{Discussion \& Conclusions}

Professor Recami says[11]: Let us recall that Riemann, as well as Clifford and later Einstein, believed that the fundamental particles of matter were the perceptible evidence of a strong local space curvature. A theory which stresses the role of space (or, rather, space-time) curvature already does exist for our whole cosmos: General Relativity, based on Einstein gravitational field equations; which are probably the most important equations of classical physical theories, together with Maxwell's electromagnetic field equations. Whilst much effort has already been made to generalize Maxwell equations, passing for examp le fro $m$ the electro magnetic field to Yang-Mills fields (so that almost all modern gauge theories are modelled on Maxwell equations), on the contrary Einstein equations have never been applied to domains different from the gravitational one. Even if they, as any differential equations, do not contain any inbuilt fundamental length: so that they can be used a priori to describe cosmoses of any size. Our first purpose is now to explore how far it is possible to apply successfully the methods of general relativity (GR), besides to the world of gravitational interactions, also to the domain of the so-called nuclear, or strong, interactions: namely, to the world of the elementary particles called hadrons. A second purpose is linked to the fact that the standard theory (QCD) of strong interactions has not yet fully explained why the hadron constituents (quarks) seem to be permanently confined in the interior of those particles; in the sense that nobody has seen up to now an isolated "free" quark, outside a hadron. So that, to explain that confinement, it has been necessary to invoke phenomenological models, such as the so-called "bag" models, in their MIT and SLAC versions for instance. The "confinement" could be explained, on the contrary, in a natural way and on the basis of a well-grounded theory like GR, if we associated with each hadron (proton, neutron, pion, ...) a particular "cosmological model".

String theory or QCD is not in a position to address the basics of cosmic structure. In understanding the basic concepts of unification or TOE, ro le of dark energy and dark matter is insignificant. Even though string theory was introduced for understanding the basics of strong interaction, its success seems to be a dilemma because of its higher dimensions and the non-coupling of the nuclear \& planck scale. Based on the proposed relations and applications, Hubble volu me or Hubble mass, can be considered as a key tool in unification as well as cosmology. From relations $(80-88)$, if it is possible to identify the atomic cosmological physical variable, then by observing the rate of change in its magnitude (on the cosmological time scale), the cosmic acceleration can be verified and thus the cosmic geometry can be confirmed from atomic and nuclear physics! Without the advancement of nano-technology or fermi-technology this may not be possible. Not only that, independent of the cosmic red shift and CMBR observations cosmic acceleration can be checked in this new direction.

In this connection, it is noticed that, proton mass is a cosmic variable and not a fundamental physical constant. Molar electron mass seems to be the planck scale mass of the proton. By this time if the observed proton is the present cosmological characteristic nuclear mass unit, then abundance of the first proton products like Hydrogen and Helium may be high. In other words, compared to the heavy atoms, light ato ms generation/abundance may be high. Thus in a very simple way, the basic and main observation of the big bang cosmology can be understood. In appendix-2, authors proposed the relation between CMBR energy density and the presently believed critical density in a growing Hubble volu me. 
Giving a fundamental importance to the 3 dimensional geometry rather than the existence of matter, it is possible to combine and study the "growing Hubble mass" and the "growing and light speed rotating primordial cosmic black hole[44]" concepts in a unified manner. Then automatically presently believed critical density comes into picture. Please recall the present concept of Hubble volume - after crossing the Hubble size, galaxies recedes with super luminal speeds. That means at the Hubble length, speed is luminal! Here the major conceptual clash is : whether the galaxy is receding or revolving (with luminal speed)? At this junction, this is a very sensitive and critical is sue to confirm and authors are working on this in different angles like basics of primordial black holes formation, basics of space-time geometry, cosmic axis of evil[44], unification and super symmetry.

Considering the proposed semi empirical relations and concepts it is possible to say that there exists a strong relation between cosmic Hubble mass and unification. Authors request the science community to kindly look into this new approach.

\section{ACKNOWLEDGEMENTS}

The first author is indebted to professor K. V. Krishna Murthy, Chairman, Institute of Scientific Research on Vedas (I-SERVE), Hyderabad, India and Shri K. V. R. S. Murthy, former scientist IICT (CSIR) Govt. of India, Director, Research and Development, I-SERVE, for their valuable guidance and great support in developing this subject.

\section{REFERENCES}

[1] Sciama, D. W. Modern Cosmology. Cambridge University Press. (1971) OCLC 6931707

[2] Hawking. S.W and George Francis Rayner Ellis. The Large Scale Structure of Space-Time. Cambridge University Press. (1973)

[3] Raine, D. J. Mach's Principle in general relativity. Royal Astronomical Society 171: 507. 1975.

[4] Bondi, et al. The Lense-Thirring Effect and Mach's Principle. Physics Letters A. Vol-228, Issue 3, 7 April 1997, Pages 121-126.

[5] R. G. Vishwakarma. A Machian Model of Dark Energy. Class.Quant.Grav. 19 (2002) 4747-4752.

[6] J. V. Narlikar. An Introduction to Cosmology. 3rd edition, Cambridge University Press.(2002)

[7] Abdus Salam. Strong Interactions, Gravitation and Cosmology. Publ. in: NATO Advanced Study Institute, Erice, June16-July 6, 1972.

[8] P. Caldirola, M. Pavsic and Recami E. Explaining the Large Numbers by a Hierarchy of Universes: A Unified Theory of Strong and Gravitational Interactions. IL Nuovo Cimento Vol. 48 B, No. 2, 11 Dec 1978.
[9] Abdus Salam. Strong Interactions, Gravitation and Cosmology. Publ. in: NATO Advanced Study Institute, Erice, June16-July 6, 1972 .

[10] Salam A, Sivaram C. Strong Gravity Approach to QCD and Confinement. Mod. Phys. Lett., 1993, v. A8(4), 321-326.

[11] Recami E. Elementary Particles as Micro-Universes, and "Strong Black-holes": A Bi-Scale Approach to Gravitational and Strong Interactions. Preprint NSF-ITP-02-04, posted in the arXives as the e-print physics/0505149, and references therein.

[12] Halpern, Paul (2004). The Great Bey ond: Higher Dimensions, Parallel Universes, and the Extraordinary Search for a Theory of Everything. Hoboken, New Jersey: John Wiley Sons, Inc.. p. 326. ISBN 0-471-46595-X.

[13] Smolin, Lee (2006). The Trouble with Physics: The Rise of String Theory, the Fall of a Science, and What Comes Next. New York: Houghton Mifflin Co.. p. 392. ISBN 0-618-55105-0.

[14] Woit, Peter (2006). Not Even Wrong - The Failure of String Theory And the Search for Unity in Physical Law. London: Jonathan Cape \&: New York: Basic Books. p. 290. ISBN 978-0-465-09275-8.

[15] E. Cremmer, B. Julia and J. Scherk. Supergravity theory in eleven dimensions, Physics Letters B76 (1978) pp 409-412.

[16] Wess, Julius; Bagger, Jonathan (1992). Super symmetry and Supergravity. Princeton University Press. pp. 260. ISBN 0691025304 .

[17] David Gross, Einstein and the search for Unification. Current science, Vol. 89, No. 12, 25 Dec 2005.

[18] Hawking S. W. A Brief History of Time. Book. Bantam Dell Publishing Group. 1988.

[19] P. J. Mohr and B.N. Taylor. CODATA recommended values of the fundamental physical constants:2006. Rev. Mod. Phys. 80 (2008) 633, Apr - Jun 2008.

[20] J. Huchara. Estimates of the Hubble Constant, 2009. Harvard-Smithsonian Center for Astrophysics. http://hubble.plot.dat

[21] W. L. Freedman et al. Final Results from the Hubble Space Telescope Key Project to Measure the Hubble Constant. The Astrophysical Journal 553 (1): 47-72.

[22] N. Jarosik et al. Seven-Year Wilson Microwave Anisotropy Probe (WMAP) Observations: Sky Maps, Systematic Errors, and Basic Results (PDF). nasa.gov. Retrieved 2010-12-02.

[23] P.A. M. Dirac. The cosmological constants. Nature, 139, 323, 1937.

[24] P. A. M. Dirac. A new basis for cosmology. Proc. Roy. Soc. A 165, 199, 1938.

[25] U. V. S. Seshavatharam and S. Lakshminarayana. Super Symmetry in Strong and Weak interactions. Int. J. Mod. Phys. E, Vol.19, No.2, (2010), p.263-280.

[26] U. V. S. Seshavatharam and S. Lakshminaray ana. SUSY and strong nuclear gravity in $(120-160) \mathrm{GeV}$ mass range. Hadronic journal, Vol-34, No 3, 2011 June, p.277-300

[27] U. V. S. Seshavatharam and S. Lakshminarayana. Nucleus in 
Strong nuclear gravity. Proceedings of the DAE Symp. on Nucl. Phys. 56 (2011) p.302

[28] U. V. S. Seshavatharam and S. Lakshminarayana. Integral charge SUSY in Strong nuclear gravity. Proceedings of the DAE Symp. on Nucl. Phys. 56 (2011) p.842

[29] Geiger $\mathrm{H}$ and Marsden E. On a diffuse reaction of the particles. Proc. Roy. Soc., Ser. A 82: 495-500, 1909.

[30] Michael O. Distler et al. The RMS Charge Radius of the Proton and Zemach Moments. Phys. Lett. B696:343-347, 2011

[31] N. Bohr. On the Constitution of Atoms and Molecules. (Part-1) Philos. Mag. 26, 11913

[32] N. Bohr. On the Constitution of Atoms and Molecules. (Part-2, Systems containing only a Single Nucleus). Philos. Mag. 26, 476, 1913

[33] U. V. S. Seshavatharam and S. Lakshminarayana. Strong nuclear gravity- a brief report. Hadronic journal. Vol. 34, No. 4, p.431, 2011.

[34] Particle Data Group (W.-M. Yao et al.), J. Phys. G 33 (2006) 1, http://pdg.bbb.gov.

[35] S. Atashbar Tehrani and A. N. Khorramian. Determination of the strong coupling constant using available experimental data. CPC(HEP $\backslash \&$ NP), 2010, 34(9): 1485-1487. Vol. 34, No. 9, Sep., 2010

[36] P. Roy Chowdhury et al. Modified Bethe-Weizsacker mass formula with isotonic shift and new driplines. Mod. Phys. Lett. A20 (2005) p.1605-1618
[37] W. D. Myers et al. Table of Nuclear Masses according to the 1994 Thomas-Fermi Model.(from nsdssd.lbl.gov)

[38] U. V. S. Seshavatharam and S. Lakshminarayana. Role of the fine structure ratio in fitting the SEMF energy constants and super heavy magic nu mbers. Hadronic journal. Vol. 35, No. 1, p.113, 2012.

[39] G. Audi and A.H. Wapstra. The 1993 atomic mass evolution.(I) Atomic mass table. Nuclear physics, A 565, 1993, p1-65.

[40] U. V. S. Seshavatharam and S. Lakshminarayana. Strong nuclear gravitational constant and the origin of nuclear planck scale. Progress in Physics, Vol-3, July, 2010.p.31-38.

[41] U.V.S. Seshavatharam and S. Lakshminarayana. Quantum Mechanics, Cosmic Acceleration and CMB Radiation. Global Journal of Science Frontier Research Vol. 12 Issue 4, p.17, 2012.

[42] U.V.S. Seshavatharam and S. Lakshmin aray ana. Accelerating universe and the expanding atom. To be appeared in the Hadronic journal.

[43] U.V.S. Seshavatharam and S. Lakshminarayana. Peculiar observations in standard cosmology and strong interactions. To be appeared in the Hadronic journal.

[44] U. V. S. Seshavatharam. The primordial cosmic black hole and the cosmic axis of evil. International Journal of Astronomy 2012, 1(2): 20-37, DOI: 10.5923/j.astronomy. 20120102.02

[45] Ebenezer Burgess. Translation of the Surya-Siddhanta. A text-book of Hindu Astronomy. Journal of the American Oriental Society, Vol. 6, 1860, pp.141- 498. 